\title{
Pipe Support Design Considerations for Hydrocarbon Facilities
}

\author{
Nirmal Surendran Menon \\ Associate Member, Institution of Mechanical Engineers (IMechE), Graduate Member, Engineers New Zealand
}

\begin{abstract}
Piping network running across a hydrocarbon facility are "arteries and veins" equivalent of a human body which carry vital fluids across the length and breadth of an operating plant so as to get it processed resulting in delivering an end user product such as Natural Gas into Liquefied Natural Gas. The pipe size ranges from small bore DN 15 (1/2") to big bore DN 1050 (42") pipes and requires adequate supporting at regular intervals so as to with stand various loads acting on the pipe or loads getting imposed on the pipe due to the fluid flowing through it.
\end{abstract}

Keywords: Pipe shoe, Piping isometric, Pipe support span, Pipe Guide, spring support, Bracing, Vitonal vibration, PWHT - Post Weld Heat Treatment, PTFE -Polytetrafluoroethylene, DN - diamètre nominal/nominal diameter, NPS - Nominal Pipe Size.

\section{Introduction}

This article focuses on defining the minimum requirements for the design of pipe supports for a pipes in hydrocarbon facilities, Liquefied Natural Gas (LNG) facilities in particular. Supports are normally for pipe line sized between DN 15 (NPS - 1/2") to DN 1050 (NPS - 42").

\section{Design Criteria}

The pipes routed throughout the facility are to be properly supported, anchored or guided to prevent undue stresses, deflection and excessive vibration and to protect both piping and connected equipment from excessive loadings which may be due to thermal, weight, steelwork deflections, wind, earthquake etc.Support points are selected to optimize load distribution and weight balance, taking into consideration available building structures to which supports can be most readily affixed. Pipes are not allowed to be supported bare on top of structural steel. This is in order to avoid excessive pipe corrosion. All pipes are therefore supported on pipe shoes. Pipe shoes are preferably shop welded where possible. Supports are designed for the piping full water hydro-test condition, except those cases when the specific gravity of the flowing medium is greater than 1.0, when the actual operating load applies. A fireproofed "catch" beam should be installed beneath piping containing flammable material where such piping is hung by rods or spring hanger type supports in fire proofed areas. Where a support from a vessel is required, a clip is incorporated in the vessel design, the welding of pipe supports directly to vessels is not allowed. Supporting pipes from other pipes are also not allowed, spring supports and hanger type supports are to be kept to a minimum, PTFE slide plates are not to be used without client approval. Supports should not encroach on headroom clearance, escape routes and lay down areas. In areas of grated flooring the support should not sit directly on the grating, but should penetrate to a structural member. Supports local to pumps are designed as to be adjustable so as to assist the field in commissioning and alignment of pump. Supports at control valve sets are to be anchored or box guided at one end and guided longitudinally at the other. Piping systems that are subjected to vibration or pulsation should be isolated from the support structure by the use of anti-vibration pads. Friction effects are to be considered at support points with the following coefficient, steel to Concrete $=0.45$, steel to Steel $=0.3$, Stainless Steel to PTFE $=0.1$

\section{Standard Pipe Support (Indication on Piping Isometrics)}

The Supports are normally indicated on isometrics as a "PIPE SUPPORT CALL OUT". The "PIPE SUPPORT CALL OUT" consists of a combination of letters and numbers, giving the type, size, length, material etc of the pipe support parts. A support for a pipe at a location can be a combination of standard support types or a special pipe support. These indications shall be written as separate items. A pipe support frame that supports multiple pipes is to be indicated only on one piping isometric drawing. For all other pipes, reference is made to the piping isometric drawing with the "PIPE SUPPORT CALL OUT" indication.

\section{1) Special Pipe Support (Indication On Piping} Isometrics)

Special pipe supports are indicated on the piping isometric. All components including support standard items will be listed on the individual special pipe support drawing. No support components will be listed on the piping isometric. A Special pipe support is indicated as SPS, where SPS indicates Special Pipe Support.

\section{2) Pipe Support Recommended Spans}

Maximum recommended support spans for Carbon Steel, Galvanized Carbon Steel, Stainless Steel and Duplex is as per Table A-1. Maximum recommended pipe support spans for CuNi and GRE pipe is as per Table A-2. In process units, common pipe racks and major facilities the maximum spacing for pipe supports is $6 \mathrm{~m}$ normally.Normally, for pipes DN 42(NPS - 24") size and smaller, the spacing between supports are selected such that the maximum deflection does not exceed $16 \mathrm{~mm}(5 / 8 ")$.

\section{3) Pipe Support Recommended Guide Spacing's}

Maximum recommended pipe support guide spacing for Carbon Steel, Stainless Steel, and Duplex \& Galvanized Carbon Steel is as per Table A-3. Maximum recommended

\section{Volume 8 Issue 2, February 2019}




\section{International Journal of Science and Research (IJSR) \\ ISSN: 2319-7064}

Impact Factor (2018): 7.426

pipe support guide spacing for $\mathrm{CuNi}$ and GRE pipe is as per Table A-4.

\section{4) Material Codes}

Under In General, the minimum allowable thickness of any structural steel is to be $10 \mathrm{~mm}$ for plates, bars and rods and $6 \mathrm{~mm}$ for rolled steel sections. Normally, pipe support steel frames are to be hot-dipped galvanized as per ASTM A123. All support frame field connections to a galvanized structure are to be welded unless otherwise noted, after site welding, repair of the galvanized structure is to be carried out as per below: For weld repair and minor damage, clean the damaged area per SSPC SP 11. Touch up with two individual coats of organic zinc-rich coating having a zinc dust loading of not less than $92 \%$ zinc in the dry film, such as ZRC "Galvilite" or ZRC "Cold Galvanizing Compound", Zinga, or equivalent. Coating Manufacturer's application instructions should be followed. Apply the specified topcoat. For major damage such as galvanized repair larger than 0.1 $\mathrm{m} 2$ as per below: Abrasive blast clean damaged area to SSPC SP 10, sand intact galvanized surface to a feathered edge. Spray-apply a coat of zinc-rich epoxy primer per Table A-5 to a dry film thickness of $60 \mu \mathrm{m}$ minimum; $100 \mu \mathrm{m}$ maximum. Apply the specified topcoat. Only galvanized fasteners should be used to connect galvanized structural members. Pipe Support material selection can be divided into 3 main groups:

Group 1: Integral welded pipe attachments forming a permanent part with the pipe by connecting welds. As per Support Material Selection Table A-6.

Group 2: Integral clamped pipe attachments forming a permanent part with the pipe by a clamped unit directly to the OD of the pipe by metal-to-metal contact and to which other parts are connected by means of welds. As per Support Material Selection Table A-7.

Group 3: Other materials - Bolts and nuts are to be coated in accordance with. Rubber for special acoustic noise suppression purpose should be made from "Vitonal" vibration plate. Temperature constancy 2000C, Hardness shores 75, Maximum allowable load $3 \mathrm{~N} / \mathrm{mm} 2$. Rubber inlay for pipe clamps and U-bolts is material grade FR80 service temperature from $-600 \mathrm{C}$ to $1500 \mathrm{C}$, shore 80 . Rubber inlay for pipe clamps and U-bolts is material grade FR80 service temperature from $-600 \mathrm{C}$ to $3500 \mathrm{C}$, shore 65 . PTFE sliding pads are from PTFE-TFLG 3124 (or approved equivalent) with an allowable contact temperature from $-350 \mathrm{C}$ to 1800C. All others, including individual indicated parts welded to table A-6 and A-7 material as per Support Material Selection Table A-8.

\section{5) Bracing of Small Bore Connections Due To Vibration and Pulsation}

Bracing of small bore branch connections DN 40 and smaller is required for all piping subject to pulsation or process induced vibration such as below:

a) Reciprocating Pumps - Connections to suction and discharge piping and equipment for reciprocating pumps (Includes first major equipment upstream and downstream of pump) b) Centrifugal pumps - Connections to pump piping within the greater of $6 \mathrm{~m}$ or 20 pipe diameters, measured along the pipe axis, from the equipment nozzle.

c) Compressors - Connections to piping within the greater of $6 \mathrm{~m}$ or 20 pipe diameters.

d) Relief Valves - Connections to PR piping within the greater of $6 \mathrm{~m}$ or 20 pipe diameters, measured along the pipe axis, from the PR device.

Bracing of small bore branch connections DN 40 and smaller is required for all piping classes $900 \#$ and above. Bracing is not required if the branch size is equal to or one size smaller than the header and is by an integrally reinforced fitting. Bracing for Thermo-wells and hydrostatic test vents is not required. The use of vibration is normally identified on the piping isometric.

\section{6) Spring Supports}

Pipeline operating at high temperature moves upwards/downwards (depending on the pipe configuration) due to thermal expansion, any rigid support provided on such a line tends to lift pipe up/down and hence remain inactive during operating conditions. In such a case a flexible support (springs) is provided which is capable of taking the load in all the operating and cold conditions. The spring supports provides continuous support during expansion or contraction of the pipe. The spring support basically employs a spring element, which can get compressed or stretch-out depending upon the thermal movement of pipe and the corresponding loads. The spring vendor normally supplies the spring units with all associated rods, turnbuckles, beam attachments, clamps, pins etc. All spring supports are to be hot dipped galvanized, spring coil to be neoprene coated.

\section{7) Pipe Shoe Selection}

Shoe standard height and length should be normally $100 \mathrm{~mm}$ high and 300mm long for pipes sizes DN500 and below, shoe height and length for pipe sizes DN600 and above should be $150 \mathrm{~mm}$ high $450 \mathrm{~mm}$ long unless noted otherwise on piping isometric. Shoe standard length in process units should be $300 \mathrm{~mm}$ and is noted on the piping isometric. This length is suitable for thermal movement up to $+/-100 \mathrm{~mm}$. Shoe standard length in pipe racks should be $450 \mathrm{~mm}$ and is noted on the piping isometric. This length is suitable for thermal movement up to $+/-175 \mathrm{~mm}$. If shims are required under pipe shoes, the shims should be welded to the support beams to ensure they remain in place.

\section{Conclusion}

All pipes supports such and supporting materials from plates, bars, rods, pipes, profiles, turn buckles, eye nuts, and bolts etc are a minimum requirement. Replacement parts should have at least equal strength and be acceptable in the total layout with respect to interference. All rods, bolts and nuts should have right-hand coarse thread. All bolts and rods should protrude through nuts a minimum of $5 \mathrm{~mm}$. All support construction should be continuously filleted welded. Other weld types should be made to avoid water penetration in seams where continuous welds are impractical; all welds should be $6 \mathrm{~mm}$ fillet. Welding symbols shown on pipe support drawings should be in accordance with the symbols 


\section{International Journal of Science and Research (IJSR) \\ ISSN: 2319-7064}

Impact Factor (2018): 7.426

from the American Welding Society (for detailed instructions, see their publication "Welding Symbols and Instructions for their use" should be referred). When welding supports to the outside of a pipe, care should be taken that welds do not burn through the pipe wall. Certain welded attachments on pipe classes that are Post Weld Heat Treated are to be highlighted on the piping isometric. Carbon steel process pipe with wall thickness $19 \mathrm{~mm}$ or greater, "WA / PWHT" to the piping isometric should be added. Welded attachments to pipe classes that are galvanized are to be avoided, but where necessary they are to be welded to the pipe Prior To Galvanizing and highlighted by adding "WA / PTG" to the piping isometric. Sloping lines are to be on shoes with the base plate in the horizontal plane having a preferred minimum height of $100 \mathrm{~mm}$. Height indicated is at the mid-point of the shoe.

\section{References}

[1] ASME B31.1 Power Piping

[2] ASME B31.3 Process Piping

[3] AS $4041 \quad$ Power Piping

[4] ASTM A 123 Standard Specification for Zinc (HotDipGalvanized) Coatings on Iron and Steel Products

[5] SSPC SP 10 Near-White Metal Blast Cleaning (NACE NO. 2)

[6] SSPC SP 11 Power Tool Cleaning to Bare Metal

[7] MSS-SP-58 Pipe Hangers and Supports - Materials, Design, and Manufacture
Table A-1:

\begin{tabular}{|c|c|c|c|c|}
\hline \multicolumn{5}{|c|}{ Maximum Recommended Pipe Support Spans } \\
\hline $\begin{array}{c}\text { Pipe Size } \\
\text { DN }\end{array}$ & $\begin{array}{c}\text { Max Span } \\
\text { M }\end{array}$ & $\begin{array}{c}\text { Pipe Size } \\
\text { DN }\end{array}$ & $\begin{array}{c}\text { Max Span } \\
\text { M }\end{array}$ \\
\hline 20 & 2.0 & & $200 \& 250$ & 8.0 \\
\hline 25 & 3.0 & & 300 & 9.0 \\
\hline $40 \& 50$ & 4.0 & & $350 \& 400$ & 10.0 \\
\hline 80 & 5.0 & & 450 to 550 & 11.0 \\
\hline 100 & 6.0 & & 600 & 12.0 \\
\hline 150 & 7.0 & & 650 to 1050 & 12.0 \\
\hline
\end{tabular}

Table A-2:

\begin{tabular}{|c|c|c|c|c|}
\hline \multicolumn{4}{|c|}{ Maximum Recommended Pipe Support Spans } \\
\hline $\begin{array}{c}\text { Pipe Size } \\
\text { DN (CuNi) }\end{array}$ & $\begin{array}{c}\text { Max Span } \\
\text { M }\end{array}$ & $\begin{array}{c}\text { Pipe Size } \\
\text { DN (GRE) }\end{array}$ & $\begin{array}{c}\text { Max Span } \\
\text { M }\end{array}$ \\
\hline & & & $50 \& 80$ & 3.0 \\
\hline 20 & 2.0 & & 100 & 4.0 \\
\hline 25 & 3.0 & & $150 \& 200$ & 5.0 \\
\hline 40 & 3.0 & & 250 to 600 & 6.0 \\
\hline
\end{tabular}

Table A-3:

Maximum Recommended Guide Spacing

\begin{tabular}{|c|c|c|c|}
\hline $\begin{array}{c}\text { Pipe Size } \\
\text { DN }\end{array}$ & $\begin{array}{c}\text { Max Span } \\
\text { M }\end{array}$ & $\begin{array}{c}\text { Pipe Size } \\
\text { DN }\end{array}$ & $\begin{array}{c}\text { Max Span } \\
\text { M }\end{array}$ \\
\hline 20 & 2.0 & 50 to 150 & 6.0 \\
\hline 25 to 40 & 3.0 & $200 \&$ Above & 12.0 \\
\hline
\end{tabular}

Table A-4:

Maximum Recommended Guide Spacing

\begin{tabular}{|c|c|c|c|c|}
\hline $\begin{array}{c}\text { Pipe Size } \\
\text { N/B }(\mathrm{CuNi})\end{array}$ & $\begin{array}{c}\text { Max Span } \\
\text { M }\end{array}$ & $\begin{array}{c}\text { Pipe Size } \\
\text { N/B (GRE) }\end{array}$ & $\begin{array}{c}\text { Max Span } \\
\text { M }\end{array}$ \\
\hline 20 & 2.0 & & 50 to 350 & 6.0 \\
\hline 25 to 40 & 3.0 & & 400 to 600 & 6.0 \\
\hline
\end{tabular}

Table A-5

\begin{tabular}{|c|c|c|c|c|c|c|c|}
\hline $\begin{array}{l}\text { Generic Product } \\
\text { Identifier }\end{array}$ & $\begin{array}{l}\text { DFT } \mu \mathrm{m} \\
\text { Min/Max } \\
\text { (ea coat) }\end{array}$ & Carboline & Hempel & International & Jotun & $\begin{array}{l}\text { Sherwin } \\
\text { Williams }\end{array}$ & Sigma \\
\hline Inorganic Zinc & $60-100$ & $\begin{array}{c}\text { CZ 11, } \\
\text { CZ11HS, or } \\
\text { CZ11 VOC }\end{array}$ & 15700 & $\begin{array}{c}\text { Interzinc } 22 \\
\text { or } 22 \mathrm{HS}\end{array}$ & Resist 86 & Zinc Clad II LV & $\begin{array}{c}\text { SigmaGuard } \\
750\end{array}$ \\
\hline Zinc Rich Epoxy & $60-110$ & Carbozinc 859 & 17380 & Interzinc 52 & Barrier 90 & Zinc Clad IV & SigmaZinc 109 \\
\hline Epoxy Primer & $70-120$ & Carboguard 888 & 15570 & $\begin{array}{c}\text { Intergard } 251 \text { or } \\
400\end{array}$ & $\begin{array}{c}\text { Jotacote } \\
\text { Universa or } \\
\text { Penguard HB }\end{array}$ & $\begin{array}{l}\text { Copoxy Shop } \\
\text { Primer }\end{array}$ & $\begin{array}{c}\text { SigmaPrime } \\
200\end{array}$ \\
\hline NSF Epoxy & $150-250$ & Carboguard 891 & 85671 & $\begin{array}{c}\text { Interseal 670HS } \\
\text { (Buff/White) }\end{array}$ & Tankguard 412 & Tank Clad HS & $\begin{array}{l}\text { SigmaGuard } \\
\text { CSF } 585\end{array}$ \\
\hline 3B Epoxy & $130-165$ & $\begin{array}{c}\text { Carbomastic } 18 \\
\text { NT }\end{array}$ & $\begin{array}{c}17630 / 3 \text { or } \\
47741 / 3\end{array}$ & Intershield 300 & $\begin{array}{c}\text { Jotacote } \\
\text { Universal }\end{array}$ & SeaGuard 6000 & $\begin{array}{c}\text { SigmaPrime } \\
200\end{array}$ \\
\hline $\begin{array}{c}\text { Temperature } \\
\text { Resistant Epoxy }\end{array}$ & $125-150$ & Thermaline 450 & 85671 & Intertherm 228 & $\begin{array}{c}\text { Tankguard } \\
\text { Storage }\end{array}$ & Epo-Phen & $\begin{array}{c}\text { Sigma } \\
\text { Phenguard } 930\end{array}$ \\
\hline $\begin{array}{c}\text { Epoxy } \\
\text { Phenolic for SS }\end{array}$ & $125-150$ & Plasite 7122 & 85671 & Intertherm 228 & $\begin{array}{c}\text { Tankguard } \\
\text { Storage }\end{array}$ & Epo-Phen & $\begin{array}{c}\text { Sigma } \\
\text { Phenguard } 940\end{array}$ \\
\hline HB Epoxy & $130-200$ & Carboguard 888 & $\begin{array}{c}45880 / 1 \text { or } \\
45080 / 3\end{array}$ & Intergard $475 \mathrm{HS}$ & $\begin{array}{l}\text { Penguard } \\
\text { Midcoat }\end{array}$ & Macropoxy 646 & $\begin{array}{c}\text { SigmaCover } \\
435\end{array}$ \\
\hline Urethane & $40-75$ & $\begin{array}{c}134 \text { or } \\
134 \mathrm{HG}\end{array}$ & $\begin{array}{l}55210 \text { or } \\
55910\end{array}$ & $\begin{array}{c}\text { Interthane } 990 \text { or } \\
990 \mathrm{HS}\end{array}$ & $\begin{array}{c}\text { HardtopAS or } \\
\text { Hardtop XP }\end{array}$ & Acrolon 218 HS & SigmaDur 550 \\
\hline Epoxy Mastic & $100-200$ & Carbomastic 15 & $45880 / 1$ & Interseal $670 \mathrm{HS}$ & $\begin{array}{l}\text { Primastic } \\
\text { Universal }\end{array}$ & Macropoxy 646 & $\begin{array}{l}\text { SigmaCover } \\
630 \text { (Alum.) }\end{array}$ \\
\hline $\begin{array}{l}\text { 100\% Solids } \\
\text { Epoxy }\end{array}$ & $300-400$ & Plasite $4550 \mathrm{~S}$ & $\begin{array}{c}35530 \text { or } \\
\text { XO } 35790\end{array}$ & $\begin{array}{c}\text { Interzone } 485 \text { or } \\
954\end{array}$ & & $\begin{array}{c}\text { Fast Clad ER } \\
\text { Epoxy }\end{array}$ & $\begin{array}{l}\text { SigmaGuard } \\
\text { CSF } 650 \text { or } \\
\text { SigmaShield } \\
905\end{array}$ \\
\hline Silicone & $20-35$ & $\begin{array}{c}4700 \text { or } \\
4700 \mathrm{VOC}\end{array}$ & 5691 & Intertherm 50 & Solvalitt & $\begin{array}{c}\text { Kem Hi Temp } \\
1200\end{array}$ & $\begin{array}{c}\text { SigmaTherm } \\
450\end{array}$ \\
\hline $\begin{array}{l}\text { Broadcast } \\
\text { Nonskid * }\end{array}$ & $*$ & 890 & 554US & Intergard $740 \mathrm{HS}$ & $\begin{array}{c}\text { Jotacoat } \\
\text { Universal }\end{array}$ & $\begin{array}{c}\text { SeaGuard } 5000 \\
\text { or }\end{array}$ & $\begin{array}{c}\text { SigmaCover } \\
456\end{array}$ \\
\hline
\end{tabular}

Volume 8 Issue 2, February 2019 
International Journal of Science and Research (IJSR)

ISSN: 2319-7064

Impact Factor (2018): 7.426

\begin{tabular}{|c|c|c|c|c|c|c|c|}
\hline $\begin{array}{l}\text { Generic Product } \\
\text { Identifier }\end{array}$ & $\begin{array}{l}\text { DFT } \mu \mathrm{m} \\
\text { Min/Max } \\
\text { (ea coat) }\end{array}$ & Carboline & Hempel & International & Jotun & $\begin{array}{c}\text { Sherwin } \\
\text { Williams }\end{array}$ & Sigma \\
\hline & & & & & & $5000 \mathrm{HS}$ & \\
\hline $\begin{array}{l}\text { Pre- Blended } \\
\text { Nonskid }\end{array}$ & $200-300$ & Gripoxy 1210 & & $\begin{array}{c}\text { Intergard 621 } \\
(\text { EK5040H } \\
\text { EBA744) } \\
\end{array}$ & & $\begin{array}{c}\text { American Safety } \\
\text { AS-250 }\end{array}$ & \\
\hline $\begin{array}{c}\text { Epoxy Glass } \\
\text { Flake } \\
\text { (Not Used } \\
\text { Onshore) }\end{array}$ & $450-750$ & 1209 & 35870 & $\begin{array}{c}\text { Interzone } 1000 \text { or } \\
505\end{array}$ & Marathon & Dura-Plate 154 & $\begin{array}{c}\text { SigmaShield } \\
905\end{array}$ \\
\hline $\begin{array}{l}\text { Shop Epoxy } \\
\text { Primer }\end{array}$ & $100-150$ & Carboguard 888 & \multirow{2}{*}{\multicolumn{3}{|c|}{ Not Available }} & $\begin{array}{c}\text { Steel Spec } \\
\text { B58 R8 } \\
\text { B58 V8 } \\
\end{array}$ & \multirow[t]{2}{*}{ See Note below } \\
\hline Epoxy Phenolic & $100-125$ & Plasite 7122 & & & & $\begin{array}{c}\text { Phenicon HS } \\
\text { 920-R-A11 } \\
\text { 700-C-685/825 }\end{array}$ & \\
\hline
\end{tabular}

Note(s): Apply in two stages - 1st layer 75-125 $\mu \mathrm{m}$, 2nd layer 50-75 $\mu \mathrm{m}$. A coat of urethane may be substituted for $2 \mathrm{nd}$ layer where required for color or aesthetics (gloss).

Table A-6

\begin{tabular}{|c|c|c|c|c|c|c|c|c|c|c|c|}
\hline \multirow{4}{*}{$\begin{array}{l}\text { Material } \\
\text { Group }\end{array}$} & \multirow{2}{*}{\multicolumn{2}{|c|}{$\begin{array}{l}\text { Service } \\
\text { Limits }\end{array}$}} & \multicolumn{7}{|c|}{ Integral welded support parts } & \multirow{3}{*}{\multicolumn{2}{|c|}{ (Other) Connecting }} \\
\hline & & & \multirow{3}{*}{ Wear pad } & \multirow{2}{*}{\multicolumn{2}{|c|}{$\begin{array}{l}\text { Steel section and plate } \\
\text { welded to }\end{array}$}} & \multirow{2}{*}{\multicolumn{2}{|c|}{ Pipe section welded to }} & \multirow{3}{*}{$\begin{array}{l}\text { Other plate or } \\
\text { steel section }\end{array}$} & \multirow{3}{*}{$\begin{array}{c}\text { Other pipe } \\
\text { section }\end{array}$} & & \\
\hline & \multirow{2}{*}{$\begin{array}{c}\text { Min } \\
0 \mathrm{C}\end{array}$} & \multirow{2}{*}{$\begin{array}{c}\text { Max } \\
0 \mathrm{C}\end{array}$} & & & & & & & & & \\
\hline & & & & Pipe & Wear plate & Pipe & Wear plate & & & Bolt & Nut \\
\hline $\mid \begin{array}{c}\text { A } \\
\text { Carbon Steel }\end{array}$ & -29 & 350 & $\mid \begin{array}{c}\text { Material as } \\
\text { per pipe class }\end{array}$ & A36 & A36 & $\begin{array}{c}\text { Material as } \\
\text { per pipe } \\
\text { class }\end{array}$ & $\begin{array}{c}\text { Material as } \\
\text { per pipe } \\
\text { class }\end{array}$ & A36 & $\begin{array}{c}\text { Material as } \\
\text { per pipe } \\
\text { class }\end{array}$ & $\begin{array}{l}\text { A193- } \\
\text { Gr.B7 }\end{array}$ & $\begin{array}{l}\text { A194- } \\
\text { Gr.2H }\end{array}$ \\
\hline$\left|\begin{array}{l}\text { B Low Temp } \\
\text { Carbon Steel }\end{array}\right|$ & -45 & 425 & $\begin{array}{c}\text { Material as } \\
\text { per pipe class }\end{array}$ & $\begin{array}{l}\text { A516-Gr65 } \\
\text { See Note } 1\end{array}$ & $\begin{array}{l}\text { A516-Gr65 } \\
\text { See Note } 1\end{array}$ & $\begin{array}{c}\text { Material as } \\
\text { per pipe } \\
\text { class }\end{array}$ & $\begin{array}{c}\text { Material as } \\
\text { per pipe } \\
\text { class }\end{array}$ & $\begin{array}{l}\text { A516-Gr65 } \\
\text { See Note } 1\end{array}$ & $\begin{array}{c}\text { Material as } \\
\text { per pipe } \\
\text { class }\end{array}$ & $\begin{array}{l}\text { A320- } \\
\text { Gr.L7 }\end{array}$ & $\begin{array}{l}\text { A194- } \\
\text { Gr.2H }\end{array}$ \\
\hline $\begin{array}{c}\text { C Stainless } \\
\text { Steel }\end{array}$ & -101 & 538 & $\begin{array}{c}\text { Material as } \\
\text { per pipe class }\end{array}$ & $\begin{array}{c}\text { A240- } \\
\text { Gr.316 (L) }\end{array}$ & $\begin{array}{c}\text { A240- } \\
\text { Gr.316 (L) }\end{array}$ & $\begin{array}{c}\text { Material as } \\
\text { per pipe } \\
\text { class }\end{array}$ & $\begin{array}{c}\text { Material as } \\
\text { per pipe } \\
\text { class }\end{array}$ & $\begin{array}{l}\text { A516-Gr65 } \\
\text { See Note } 1\end{array}$ & N/A & $\begin{array}{l}\text { A320- } \\
\text { Gr.L7 }\end{array}$ & $\begin{array}{l}\text { A194- } \\
\text { Gr.G4 }\end{array}$ \\
\hline $\begin{array}{l}\text { D Duplex } \\
\text { DSS }\end{array}$ & -51 & 280 & $\begin{array}{c}\text { Material as } \\
\text { per pipe class }\end{array}$ & $\begin{array}{c}\text { A240- } \\
\text { Gr.316 (L) }\end{array}$ & $\begin{array}{c}\text { A240- } \\
\text { Gr.316 (L) }\end{array}$ & $\begin{array}{c}\text { Material as } \\
\text { per pipe } \\
\text { class }\end{array}$ & $\begin{array}{c}\text { Material as } \\
\text { per pipe } \\
\text { class }\end{array}$ & $\begin{array}{l}\text { A516-Gr65 } \\
\text { See Note } 1\end{array}$ & N/A & $\begin{array}{l}\text { A320- } \\
\text { Gr.L7 }\end{array}$ & $\begin{array}{l}\text { A194- } \\
\text { Gr.G4 }\end{array}$ \\
\hline
\end{tabular}

Note(s): 1. Material required to be normalized and impact tested at $-45^{0} \mathrm{C}$.

Table A-7

\begin{tabular}{|c|c|c|c|c|c|c|c|c|c|c|}
\hline \multirow{4}{*}{$\begin{array}{l}\text { Material } \\
\text { Group }\end{array}$} & \multirow{2}{*}{\multicolumn{2}{|c|}{$\begin{array}{l}\text { Service } \\
\text { Limits }\end{array}$}} & \multicolumn{6}{|c|}{ Support part materials } & \multirow{3}{*}{\multicolumn{2}{|c|}{$\begin{array}{l}\text { (Others) } \\
\text { Connecting }\end{array}$}} \\
\hline & & & \multicolumn{3}{|c|}{ Support clamp } & \multirow{3}{*}{ U-Bolt } & \multirow{3}{*}{$\begin{array}{c}\text { Plate or steel } \\
\text { section }\end{array}$} & \multirow{3}{*}{ Pipe section } & & \\
\hline & \multirow{2}{*}{$\begin{array}{c}\text { Min } \\
\text { OC }\end{array}$} & \multirow{2}{*}{$\begin{array}{c}\text { Max } \\
\text { OC }\end{array}$} & \multirow{2}{*}{ Steel strip } & \multirow{2}{*}{ Bolt } & \multirow{2}{*}{ Nut } & & & & & \\
\hline & & & & & & & & & Bolts & Nuts \\
\hline $\begin{array}{c}\text { E Carbon } \\
\text { Steel } \\
\end{array}$ & -29 & 425 & A36 & A193 & A194-Gr.2H & $\begin{array}{c}\text { A36 } \\
\text { (See note 2) } \\
\end{array}$ & A36 & A106- & $\begin{array}{c}\text { A193- } \\
\text { Gr.7 } \\
\end{array}$ & $\begin{array}{l}\text { A194- } \\
\text { Gr.2H } \\
\end{array}$ \\
\hline $\begin{array}{c}\text { F Low Temp } \\
\text { Carbon } \\
\text { Steel } \\
\end{array}$ & -45 & 425 & \begin{tabular}{|c|} 
A516-Gr65 \\
(See note 1)
\end{tabular} & A320-Gr.L7 & A194-Gr.2H & $\begin{array}{c}\text { A516-Gr65 } \\
\text { (See notes } \\
1 \& 2) \\
\end{array}$ & $\begin{array}{l}\text { A516-Gr65 } \\
\text { (See note 1) }\end{array}$ & $\begin{array}{c}\text { Material as } \\
\text { per pipe class }\end{array}$ & $\begin{array}{l}\text { A320- } \\
\text { Gr.L7 }\end{array}$ & $\begin{array}{l}\text { A194- } \\
\text { Gr.2H }\end{array}$ \\
\hline $\begin{array}{c}\text { G \& H } \\
\text { Stainless Steel } \\
\text { \& Duplex DSS }\end{array}$ & -101 & 538 & $\begin{array}{c}\text { A240- Gr.316 } \\
\text { (L) }\end{array}$ & A320-Gr.L7 & A194-Gr.2H & A276 Gr.316 & $\begin{array}{c}\text { A240- Gr.316 } \\
\text { (L) }\end{array}$ & $\begin{array}{c}\text { Material as } \\
\text { per pipe class }\end{array}$ & $\begin{array}{l}\text { A320- } \\
\text { Gr.L7 }\end{array}$ & $\begin{array}{l}\text { A194- } \\
\text { Gr.2H }\end{array}$ \\
\hline
\end{tabular}

Notes:

1. Material required to be normalized and impact tested at $-45^{\circ} \mathrm{C}$.

2. U-Bolt to be hot-dipped galvanized with a neoprene covering.

Table A-8

\begin{tabular}{|c|c|c|c|c|c|c|c|c|}
\hline $\begin{array}{c}\text { Material } \\
\text { Group }\end{array}$ & $\begin{array}{c}\text { Plate, Bar, } \\
\text { Clamp }\end{array}$ & $\begin{array}{c}\text { Steel Section, } \\
\text { Rod, Pin }\end{array}$ & Pipe & Forged Steel & Bearing plate for PTFE & PTFE & Bolt & Nut \\
\hline W & $\begin{array}{c}\text { ASTM } \\
\text { A36 }\end{array}$ & ASTM A36 & $\begin{array}{c}\text { ASTM } \\
\text { A106 Gr.B }\end{array}$ & ASTM A668 Gr.C & A240-Gr.304 & PTFE & $\begin{array}{c}\text { A193 } \\
\text { Gr.7 }\end{array}$ & $\begin{array}{c}\text { A194 } \\
\text { Gr,.2H }\end{array}$ \\
\hline
\end{tabular}

\section{Volume 8 Issue 2, February 2019 www.ijsr.net}

